\title{
Prevalence of Neuropathic Pain in Rheumatic Disorders: Association With Disease Activity, Functional Status and Quality of Life
}

\author{
Yeşim GARİP, ${ }^{1}$ Filiz ESER, ${ }^{2}$ Ayşegül KILIÇARSLAN, ${ }^{2}$ Hatice BODUR ${ }^{2}$ \\ ${ }^{1}$ Department of Physical Medicine and Rehabilitation, Başak Medical Center, Ankara, Turkey \\ ${ }^{2}$ Department of Physical Medicine and Rehabilitation, Ankara Numune Training and Research Hospital, Ankara, Turkey
}

\begin{abstract}
Objectives: This study aims to investigate neuropathic pain in rheumatologic disorders including rheumatoid arthritis (RA), ankylosing spondylitis (AS), and osteoarthritis (OA) using PainDETECT and to determine its effect on the quality of life in terms of disease activity, functional status, social and emotional functioning.

Patients and methods: A total of 150 patients ( 66 males, 84 females; mean age $48.44 \pm 12.22$ years; range 25 to 65 years) were included in the study. Of these patients, 50 had OA, 50 had RA, and 50 had AS. Control group consisted of 50 healthy subjects ( 20 males, 30 females; mean age $48.36 \pm 12.68$ years; range 25 to 65 years). OA severity was evaluated by Western Ontario and McMasters Universities Index of Osteoarthritis. In RA patients, Disease Activity Score-28 was used for measuring disease activity, and Stanford Health Assessment Questionnaire for functional status. In AS patients, disease activity was assessed by Bath Ankylosing Spondylitis Disease Activity Index and functional status by Bath Ankylosing Spondylitis Functional Index. Neuropathic pain was determined by PainDETECT questionnaire and quality of life by Nottingham Health Profile.

Results: Prevalence of neuropathic pain was $44 \%$ in OA, $28 \%$ in AS, and $18 \%$ in RA patients. Compared with control patients, prevalence was higher in OA [Odds ratio $=12.4695 \%$ confidence interval $(3.89-39.85)](p=0.00)$ and AS patients [Odds ratio $=4.4795 \%$ confidence interval $(1.36-14.76)$ ] $(p=0.009)$. In OA patients, PainDETECT was correlated with Western Ontario and McMasters Universities Index of Osteoarthritis $(p=0.00)$. In all of the patient groups, PainDETECT was correlated with Nottingham Health Profile $(p=0.00)$. Physical mobility subgroup showed the strongest correlation with PainDETECT.

Conclusion: Our study demonstrated that neuropathic pain is strongly associated with quality of life in terms of physical mobility, energy, sleep, and social and emotional functions. The disease with highest prevalence of neuropathic pain was OA. A better understanding of neuropathic pain mechanisms in rheumatic diseases will help us find more effective treatment strategies.

Keywords: Ankylosing spondylitis; pain; quality of life; rheumatoid arthritis.
\end{abstract}

Chronic pain is classified as nociceptive (inflammatory) pain and neuropathic pain. Nociceptive pain is caused by stimulation of nociceptors through chronic inflammation. ${ }^{1}$ Neuropathic pain is defined as "pain caused by a primary lesion or dysfunction in the nervous system" by International Association for the Study of Pain. ${ }^{2}$ Peripheral and central sensitization, inhibition of descending pain inhibitory systems, functional changes in autonomic nervous system and neurotransmitters are important factors in neuropathic pain. ${ }^{3}$ Patients describe neuropathic pain as burning, painful, cold or electric shocks and possibly associate it with tingling, pins and needles, numbness or itching. ${ }^{4}$

Prevalence of neuropathic pain is not exactly known. Best current estimates of the population prevalence of neuropathic pain come from studies using validated screening tools that detect pain with possible neuropathic features. ${ }^{5}$ Previous studies have shown that prevalence of neuropathic pain is around $7-8 \%$ in the general population. ${ }^{6}$ There are only a few studies that investigated the prevalence of neuropathic pain in rheumatic diseases in the literature. 
Neuropathic pain must be correctly diagnosed for optimal treatment, since neuropathic and non-neuropathic pain require different treatment strategies. Several screening tools have been developed to differentiate neuropathic pain from non-neuropathic pain. ${ }^{7}$ They also serve as a good clinical record for follow-up of the patients. ${ }^{4}$

PainDETECT is a simple, self-administered, useful screening questionnaire, which was designed to evaluate neuropathic signs and symptoms without physical examination. ${ }^{8}$ It was developed by Freynhagen et al. ${ }^{9}$ to identify neuropathic components in patients with back pain. It was found to be a reliable tool with high sensitivity, specificity, and positive predictive accuracy.

Neuropathic pain is not a single disease, but a syndrome caused by a range of different diseases and lesions, which has a considerable impact on the quality of life (QoL). ${ }^{8}$ This study aims to investigate neuropathic pain in rheumatologic disorders including rheumatoid arthritis (RA), ankylosing spondylitis (AS) and osteoarthritis (OA) using PainDETECT and to determine its effect on the QoL in terms of disease activity, functional status, social and emotional functioning.

\section{PATIENTS AND METHODS}

A total of 150 patients (66 males, 84 females; mean age $48.44 \pm 12.22$ years; range 25 to 65 years) [50 OA patients (21 males, 29 females; mean age $50.9 \pm 5.12$ years; range 41 to 65 years), 50 RA patients (10 males, 40 females; mean age $55.64 \pm 13.47$ years; range 25 to 65 years), and 50 AS patients (35 males, 15 females; mean age $38.78 \pm 9.59$ years; range 25 to 60 years)] followed consecutively at outpatient physical medicine and rehabilitation clinic of Numune Training and Research Hospital between February and April 2014 were enrolled in the study.

Control group consisted of 50 healthy subjects (20 males, 30 females; mean age 48.36 \pm 12.68 years; range 25 to 65 years). Exclusion criteria were neurological diseases such as multiple sclerosis, cerebrovascular disorders, and peripheral neuropathy and endocrine diseases such as diabetes mellitus and thyroid disorders. Patient information regarding age, sex, and duration of the disease was recorded. All of the patients were informed about the study, and their written informed consent was taken. Medical Research Ethics Committee of the training and research hospital approved the study protocol. The study conforms to the provisions of the World Medical Association's Declaration of Helsinki.

Osteoarthritis patients were diagnosed as knee OA according to the American College of Rheumatology for classification of knee OA. ${ }^{10}$ All patients had knee pain for more than one month. Severity of OA was evaluated by using Western Ontario and McMasters Universities Index of Osteoarthritis (WOMAC). Level of pain was assessed by using WOMAC A, stiffness by WOMAC B, and functional impairment by WOMAC C. ${ }^{11}$

Rheumatoid arthritis patients had a positive diagnosis of RA according to the diagnostic criteria of American College of Rheumatology. ${ }^{12}$ Number of swollen and tender joints, erythrocyte sedimentation rate $(\mathrm{mm} /$ hour) and C-reactive protein $(\mathrm{mg} / \mathrm{dL})$ levels were measured. The quadrivariate Disease Activity Score-28 was used for measuring disease activity. Values under 3.2 were accepted as low, in the range of 3.2-5.1 as intermediate, and over 5.1 as high disease activity. ${ }^{13}$ Functional status was determined by using the Stanford Health Assessment Questionnaire. ${ }^{14}$

Ankylosing spondylitis patients fulfilled the modified New York criteria. ${ }^{15}$ Patients were evaluated by using Assessment of SpondyloArthritis International Society recommendations for core outcome domains for the assessment in AS. ${ }^{16}$ Erythrocyte sedimentation rate and C-reactive protein levels were measured. Disease activity was evaluated by using Bath Ankylosing Spondylitis Disease Activity Index. ${ }^{17}$ The Bath Ankylosing Spondylitis Functional Index was used for determining functional status. ${ }^{18}$ QoL of the patients was assessed by using Nottingham Health Profile (NHP). ${ }^{19}$

Presence of neuropathic pain was determined by using PainDETECT questionnaire. $^{8}$ The PainDETECT is a simple, self-administered questionnaire, which was developed to detect neuropathic pain in patients with chronic pain. It comprises four dimensions. 
The first dimension contains three items that assess intensity of pain at the moment, the average and the maximum pain intensity during the past four weeks. In the second dimension, patients are asked to mark one of the four graphs that describe their pain course patterns. The third dimension consists of a sensory map representing homunculus along with questions asking to mark the pain zone, a dichotomous item about the presence of radiating pain and showing the direction of radiating pain with an arrow. In the fourth dimension, there are seven questions about the following sensations: burning, tingling or prickling, allodynia, pain attacks, temperature evoked pain, numbness, and pressure-evoked pain. The final score is obtained by summing up the scores of the last three sections with a total score of -1 to 38 . Scores $\leq 12$ indicate that a neuropathic pain component is unlikely, and scores $\geq 19$ indicate that neuropathic component is very likely to be present. Scores between 12 and 19 suggest that the result is unclear. Its adaptation to Turkish language and validation were proven by Alkan et al. $^{8}$

\section{Statistical analysis}

Descriptive statistics [mean, median, standard deviation (SD), minimum, maximum and frequencies] were used for assessing the demographics and clinical parameters. Chisquared test was used to compare groups of categorical variables. Analysis of correlation was made with Pearson's correlation coefficient.

\begin{tabular}{|c|c|c|}
\hline & Mean \pm SD & Min.-Max. \\
\hline Age (years) & $50.9 \pm 5.1$ & $41-65$ \\
\hline Pain (VAS, $10 \mathrm{~cm}$ ) & $4.7 \pm 2.6$ & $0-10$ \\
\hline PainDETECT & $12.8 \pm 10.0$ & $-1-38$ \\
\hline WOMAC A & $11.9 \pm 4.7$ & $1-20$ \\
\hline WOMAC B & $4.7 \pm 1.8$ & $0-8$ \\
\hline WOMAC C & $43.3 \pm 14.3$ & $10-70$ \\
\hline WOMAC total & $59.2 \pm 19.5$ & $11-92$ \\
\hline NHP-pain & $44.6 \pm 33.1$ & $0-100$ \\
\hline NHP-physical mobility & $39.3 \pm 29.6$ & $0-100$ \\
\hline NHP-energy & $48.0 \pm 42.0$ & $0-100$ \\
\hline NHP-sleep & $40.6 \pm 34.6$ & $0-100$ \\
\hline NHP-social isolation & $37.0 \pm 34.3$ & $0-100$ \\
\hline NHP-emotional reactions & $46.5 \pm 37.0$ & $0-100$ \\
\hline
\end{tabular}

A value of $p<0.05$ was considered statistically significant. All analyses were performed using IBM SPSS version 21.0 software program (IBM Corporation, Armonk, New York. USA).

\section{RESULTS}

In OA patients, mean score was $11.86 \pm 4.66$ for WOMAC A, 4.72 \pm 1.81 for WOMAC B, $43.28 \pm 14.29$ for WOMAC C, and $59.24 \pm 19.48$ for WOMAC total. The mean QoL scores of the patients were $44.56 \pm 33.07,39.25 \pm 29.62$, $48.00 \pm 41.99,40.60 \pm 34.75,37.00 \pm 34.27$, and $46.5 \pm 37.02$ in the pain, physical mobility, energy, sleep, social isolation and emotional reactions subgroups of NHP, respectively. Demographic and clinical data of the patients are summarized in Table 1.

In RA patients, mean score was $2.81 \pm 1.16$ for Disease Activity Score-28, and 1.44 \pm 1.55 for Health Assessment Questionnaire. The mean QoL scores of the patients were $44.14 \pm 34.34$, $34.00 \pm 29.88, \quad 49.00 \pm 43.44, \quad 35.6 \pm 37.32$, $32.00 \pm 35.23$, and $40.25 \pm 36.62$ in the pain, physical mobility, energy, sleep, social isolation and emotional reactions subgroups of NHP, respectively. Demographic data and clinical characteristics of the patients are summarized in Table 2.

Table 2. Demographic and clinical data of patients with rheumatoid arthritis

\begin{tabular}{lcc}
\hline & Mean \pm SD & Min.-Max. \\
\hline Age (years) & $55.6 \pm 13.5$ & $25-65$ \\
Disease duration (year) & $13.8 \pm 9.4$ & $1-40$ \\
Pain (VAS, $10 \mathrm{~cm}$ ) & $3.9 \pm 2.9$ & $0-10$ \\
PainDETECT & $8.6 \pm 7.3$ & $-1-27$ \\
ESR (mm/hour) & $20.6 \pm 18.2$ & $2-103$ \\
CRP (mg/dL) & $12.6 \pm 16.7$ & $0.28-89$ \\
Number of swollen joints & $0.3 \pm 1.0$ & $0-5$ \\
Number of tender joints & $2.7 \pm 5.1$ & $0-20$ \\
DAS28 & $2.8 \pm 1.2$ & $0.68-5.68$ \\
HAQ & $1.4 \pm 1.6$ & $0-6$ \\
NHP-pain & $44.1 \pm 34.3$ & $0-100$ \\
NHP-physical mobility & $34.0 \pm 29.9$ & $0-100$ \\
NHP-energy & $49.0 \pm 43.4$ & $0-100$ \\
NHP-sleep & $35.6 \pm 37.3$ & $0-100$ \\
NHP-social isolation & $32.0 \pm 35.2$ & $0-100$ \\
NHP-emotional reactions & $40.3 \pm 36.6$ & $0-100$ \\
\hline SD: Standard deviation; Min.: Minimum; Max.: Maximum; VAS: Visual \\
analog scale; ESR: Erythrocyte sedimentation rate; CRP: C-reactive \\
protein; DAS28: Disease Activity Score-28; HAQ: Stanford Health \\
Assessment Questionnaire; NHP: Nothingham Health Profile. \\
\hline
\end{tabular}


Table 3. Demographic and clinical data of patients with ankylosing spondylitis

\begin{tabular}{lcc}
\hline & Mean \pm SD & Min.-Max. \\
\hline Age (years) & $38.8 \pm 9.6$ & $25-60$ \\
Disease duration (year) & $10.0 \pm 7.5$ & $1-36$ \\
Pain (VAS, 10 cm) & $5.6 \pm 2.0$ & $2-10$ \\
PainDETECT & $12.1 \pm 9.6$ & $-1-37$ \\
ESR (mm/hour) & $16.9 \pm 16.1$ & $2-77$ \\
CRP (mg/dL) & $12.8 \pm 12.1$ & $0.2-55$ \\
BASDAI & $3.6 \pm 2.4$ & $0-9.1$ \\
BASFI & $2.7 \pm 2.6$ & $0-8.8$ \\
NHP-pain & $41.1 \pm 32.4$ & $0-100$ \\
NHP-physical mobility & $35.7 \pm 29.7$ & $0-100$ \\
NHP-energy & $45.0 \pm 41.3$ & $0-100$ \\
NHP-sleep & $38.1 \pm 33.5$ & $0-100$ \\
NHP-social isolation & $35.1 \pm 32.9$ & $0-100$ \\
NHP-emotional reactions & $42.5 \pm 36.1$ & $0-100$ \\
\hline SD: Standard deviation; Min.: Minimum; Max.: Maximum; VAS: Visual \\
analog scale; ESR: Erythrocyte sedimentation rate; CRP: C-reactive \\
protein; BASDAI: Bath Ankylosing Spondylitis Disease Activity Index; \\
BASFI: Bath Ankylosing Spondylitis Functional Index; NHP: Nothingham \\
Health Profile. \\
\hline
\end{tabular}

In AS patients, mean score was $3.55 \pm 2.36$ for Bath Ankylosing Spondylitis Disease Activity Index, and 2.72 \pm 2.55 for Bath Ankylosing Spondylitis Functional Index. The mean QoL scores of the patients were $41.14 \pm 32.39$, $35.67 \pm 29.69, \quad 45.00 \pm 41.27, \quad 38.13 \pm 33.53$, $35.07 \pm 32.85$, and $42.50 \pm 36.09$ in the pain, physical mobility, energy, sleep, social isolation and emotional reactions subgroups of NHP, respectively. Demographic and clinical data for AS patients are summarized in Table 3 .

Mean PainDETECT score of OA patients was $12.81 \pm 9.99$ (Table 2). PainDETECT was found to be $\geq 19$ in 22 patients (44\%). Mean PainDETECT score of RA patients was 8.57 \pm 7.34 (Table 2). PainDETECT was found to be $\geq 19$ in nine patients (18\%). Mean PainDETECT score of AS patients was 12.06 \pm 9.61 (Table 2). PainDETECT was found to be $\geq 19$ in14 patients (28\%).

According to PainDETECT, the prevalence of neuropathic pain was $44 \%$ in OA, $28 \%$ in AS, $18 \%$ in RA patients, and $8 \%$ in controls.
The prevalence of neuropathic pain was higher in patients with OA lodds ratio $=12.46$ 95\% confidence interval (3.89-39.85)] $(p=0.00)$ and in patients with AS (odds ratio $=4.4795 \%$ confidence interval (1.36-14.76)] $(p=0.009)$, compared with controls (Table 4). There was no statistically significant difference between neuropathic pain prevalence of RA and control group [odds ratio $=2.5295 \%$ confidence interval $(0.72-8.82)]$ $(p=0.14)$ (Table 4).

In OA patients, PainDETECT was correlated with WOMAC A, B, C and WOMAC total $(\mathrm{r}=0.88,0.74,0.95$, and 0.96 , respectively) $(p=0.00)$ and physical mobility, pain, emotional reactions, sleep, social isolation, and energy subgroups of NHP ( $\mathrm{r}=0.77,0.76,0.75,0.69$, 0.66 , and 0.63 , respectively) $(p=0.00)$. Analyses of correlation coefficients revealed that the strongest relation of PainDETECT was with physical mobility subgroup of NHP.

In AS patients, there was no significant correlation between PainDETECT and Bath Ankylosing Spondylitis Disease Activity Index, and Bath Ankylosing Spondylitis Functional Index $(p=0.18, r=-0.19$ and $p=0.58, r=-0.08$, respectively). PainDETECT was related with physical mobility, emotional reactions, social isolation, sleep, energy, and pain subgroups of NHP at high levels $(r=0.82,0.81,0.80,0.79$, 0.72 , and 0.68 , respectively) $(p=0.00)$. Analyses of correlation coefficients revealed that the strongest relation of PainDETECT was with physical mobility subgroup of NHP. When the correlation coefficients were analyzed, PainDETECT showed the highest correlation with physical mobility.

In RA patients, there was no significant correlation between PainDETECT and Disease Activity Score-28, and Health Assessment Questionnaire $(p=0.18, \quad r=0.17$ and $p=0.19$, $r=0.18$, respectively). PainDETECT was correlated with physical mobility, pain, emotional

Table 4. Prevalence of neuropathic pain in patients with osteoarthritis, ankylosing spondylitis and rheumatoid arthritis

\begin{tabular}{lcccc}
\hline & $\mathrm{n}$ & $\%$ & Difference $(95 \% \mathrm{CI})$ & $p$ \\
\hline Osteoarthritis patients & 22 & 44 & $12.46(3.89-39.85)$ & $0.00^{* * *}$ \\
Ankylosing spondylitis patients & 14 & 28 & $2.52(0.72-8.82)$ & $0.009^{*}$ \\
Rheumatoid arthritis patients & 9 & 18 & $4.47(1.36-14.76)$ & 0.14 \\
\hline CI: Confidence interval; ${ }^{*} \mathrm{p}<0.05$ (significant), ${ }^{* *} \mathrm{p}<0.01$ (highly significant). & \\
\hline
\end{tabular}


reactions, sleep, social isolation, and energy subgroups of NHP $(\mathrm{r}=0.65,0.64,0.59,0.56$, 0.55 , and 0.54 , respectively) $(p=0.00)$. The strongest relation of PainDETECT was with physical mobility subgroup of NHP, according to the correlation coefficients.

\section{DISCUSSION}

Recent epidemiologic surveys of the general population have suggested that the prevalence of neuropathic pain is $7-8 \% .^{20,21}$ To our knowledge, there are few studies in the literature investigating the prevalence of neuropathic pain in patients with rheumatic disorders. In our study, the rate of neuropathic pain was shown as $44 \%$ in patients with knee OA. In a 92-case crosssectional study from Japan, the neuropathic pain frequency in patients with knee $\mathrm{OA}$ was reported to be $5.4 \% .{ }^{22}$ Hochman et al. ${ }^{23}$ reported prevalence of neuropathic pain symptoms as $28 \%$ among older adults with chronic symptomatic knee OA in Canada. They suggested that, in addition to nociceptive mechanisms, neuropathic mechanisms might contribute to chronic pain in patients with OA. Also, previous studies indicated both nociceptive and neuropathic pain in rat models which had surgically and mono-iodoacetate-induced OA. ${ }^{24,25}$ There are possible mechanisms that may explain neuropathic pain in OA. According to McDougall et al., ${ }^{26}$ subclinical damage to peripheral nerves may be associated with neuropathic pain in knee OA. In early phases, synovitis produces joint fluid and stimulates free nerve endings in synovium. In late phases of OA, a decreased amount of joint fluid and destruction of the osteochondral junction may induce injury to the nerve endings in this area. ${ }^{22}$ Along damaged nerves, increased ectopic activity may occur and contribute to ongoing pain. ${ }^{23}$

PainDETECT was demonstrated to be associated with all subgroups of WOMAC in OA patients. This association was found previously in the study of Hochman et al. ${ }^{27}$ They reported that neuropathic pain symptoms were significantly associated with greater OA severity.

In our study, prevalence of neuropathic pain was $18 \%$ in RA patients. Perrot et al. ${ }^{28}$ detected the frequency of neuropathic pain in 37.5\% of RA patients, where the neuropathic pain was assessed by using Douleur Neuropathique en 4 Questions. In our study, pain levels did not reflect disease activity and functional status in RA. On the contrary, in the study of Perrot, ${ }^{28}$ severity of pain was found to be correlated with disease activity. Different results may be due to our patient selection criteria. We excluded the patients with neurological involvement such as nerve entrapment syndromes, mononeuritis multiplex, and atlantoaxial subluxation.

The prevalence of neuropathic pain was higher (28\%) in patients with AS, compared with controls. To the best of our knowledge, ours is the first study to examine the frequency of neuropathic pain in AS in the literature. There is some evidence about neuropathic pain in AS. Wu et al. ${ }^{29}$ states that pain in AS is a mixed pain condition that includes a neuropathic pain component. They explained the neuropathic pain mechanism in AS with 'inflammatory radiculopathy'. Local inflammation of the axial spine in AS affects dorsal ramus branches that innervate zygapophyseal joints and periosteum of the vertebral arch, and cause the 'inflammatory radiculopathy' via the action of inflammatory mediators, even without mechanical compression. The relationship of neuropathic pain severity with disease activity and functional impairment has not been studied previously, to our knowledge, in AS population. We found no association between the severity of neuropathic pain, and disease activity and functional status.

To our knowledge, this is the first study to assess the impact of neuropathic pain on $\mathrm{QoL}$ domains in rheumatic diseases. We found strong associations between the severity of neuropathic pain and QoL domains including pain, physical mobility, energy, sleep, and social and emotional functions in the patients with OA, RA and AS. Physical mobility subgroup showed the strongest correlation with PainDETECT. These findings show that neuropathic pain has major negative influence on QoL in terms of physical mobility.

This study demonstrates several important observations regarding neuropathic pain in rheumatic disorders. The first important finding was that the disease with the highest prevalence of neuropathic pain was OA, and the severity of neuropathic pain was associated with the severity of OA, confirming the previous data in the literature. Second, neuropathic pain had a 
negative impact on QoL in terms of pain, physical mobility, energy, sleep, and social and emotional functions in patients with OA, RA and AS.

Since neuropathic and non-neuropathic pain require different treatment strategies, neuropathic pain must be diagnosed by using validated screening tools. A better understanding of neuropathic pain mechanisms in rheumatic diseases will provide a more targeted approach to pain treatment.

\section{Declaration of conflicting interests}

The authors declared no conflicts of interest with respect to the authorship and/or publication of this article.

\section{Funding}

The authors received no financial support for the research and/or authorship of this article.

\section{REFERENCES}

1. Siddall PJ, McClelland JM, Rutkowski SB, Cousins MJ. A longitudinal study of the prevalence and characteristics of pain in the first 5 years following spinal cord injury. Pain 2003;103:249-57.

2. Jensen TS, Baron R, Haanpää M, Kalso E, Loeser JD, Rice AS, et al. A new definition of neuropathic pain. Pain 2011;152:2204-5.

3. Berker E, Dinçer N. Chronic pain and rehabilitation. [Article in Turkish] Agri 2005;17:10-6.

4. Votrubec M, Thong I. Neuropathic pain--a management update. Aust Fam Physician 2013;42:92-7.

5. Torrance N, Ferguson JA, Afolabi E, Bennett MI, Serpell MG, Dunn KM, et al. Neuropathic pain in the community: more under-treated than refractory? Pain 2013;154:690-9.

6. Gauffin J, Hankama T, Kautiainen H, Hannonen P, Haanpää M. Neuropathic pain and use of PainDETECT in patients with fibromyalgia: a cohort study. BMC Neurol 2013;13:21.

7. Sommer C, Richter $H$, Rogausch JP, Frettlöh J, Lungenhausen M, Maier C. A modified score to identify and discriminate neuropathic pain: a study on the German version of the Neuropathic Pain Symptom Inventory (NPSI). BMC Neurol 2011;11:104.

8. Alkan H, Ardic F, Erdogan C, Sahin F, Sarsan A, Findikoglu G. Turkish version of the painDETECT questionnaire in the assessment of neuropathic pain: a validity and reliability study. Pain Med 2013;14:1933-43.

9. Freynhagen R, Baron R, Gockel U, Tölle TR. painDETECT: a new screening questionnaire to identify neuropathic components in patients with back pain. Curr Med Res Opin 2006;22:1911-20.

10. Altman R, Asch E, Bloch D, Bole G, Borenstein $\mathrm{D}$, Brandt $\mathrm{K}$, et al. Development of criteria for the classification and reporting of osteoarthritis. Classification of osteoarthritis of the knee. Diagnostic and Therapeutic Criteria Committee of the American Rheumatism Association. Arthritis Rheum 1986;29:1039-49.

11. Tüzün EH, Eker L, Aytar A, Daşkapan A, Bayramoğlu M. Acceptability, reliability, validity and responsiveness of the Turkish version of WOMAC osteoarthritis index. Osteoarthritis Cartilage 2005;13:28-33.

12. Arnett FC, Edworthy SM, Bloch DA, McShane DJ, Fries JF, Cooper NS, et al. The American Rheumatism Association 1987 revised criteria for the classification of rheumatoid arthritis. Arthritis Rheum 1988;31:315-24.

13. Prevoo ML, van 't Hof MA, Kuper HH, van Leeuwen MA, van de Putte LB, van Riel PL. Modified disease activity scores that include twenty-eight-joint counts. Development and validation in a prospective longitudinal study of patients with rheumatoid arthritis. Arthritis Rheum 1995;38:44-8.

14. Küçükdeveci AA, Sahin $H$, Ataman S, Griffiths $\mathrm{B}$, Tennant A. Issues in cross-cultural validity: example from the adaptation, reliability, and validity testing of a Turkish version of the Stanford Health Assessment Questionnaire. Arthritis Rheum 2004;51:14-9.

15. Moll JM. New criteria for the diagnosis of ankylosing spondylitis. Scand J Rheumatol Suppl 1987;65:12-24.

16. Sieper J, Rudwaleit M, Baraliakos X, Brandt J, Braun J, Burgos-Vargas $\mathrm{R}$, et al. The Assessment of SpondyloArthritis international Society (ASAS) handbook: a guide to assess spondyloarthritis. Ann Rheum Dis 2009;68:1-44.

17. Akkoc Y, Karatepe AG, Akar S, Kirazli Y, Akkoc N. A Turkish version of the Bath Ankylosing Spondylitis Disease Activity Index: reliability and validity. Rheumatol Int 2005;25:280-4.

18. Ozer HT, Sarpel T, Gulek B, Alparslan ZN, Erken E. The Turkish version of the Bath Ankylosing Spondylitis Functional Index: reliability and validity. Clin Rheumatol 2005;24:123-8.

19. Kücükdeveci AA, McKenna SP, Kutlay S, Gürsel Y, Whalley D, Arasil T. The development and psychometric assessment of the Turkish version of the Nottingham Health Profile. Int $\mathrm{J}$ Rehabil Res 2000;23:31-8.

20. Torrance N, Smith BH, Bennett MI, Lee AJ. The epidemiology of chronic pain of predominantly neuropathic origin. Results from a general population survey. J Pain 2006;7:281-9.

21. Bouhassira D, Lantéri-Minet $\mathrm{M}$, Attal N, Laurent B, Touboul C. Prevalence of chronic pain with neuropathic characteristics in the general population. Pain 2008;136:380-7. 
22. Ohtori S, Orita S, Yamashita M, Ishikawa T, Ito T, Shigemura $\mathrm{T}$, et al. Existence of a neuropathic pain component in patients with osteoarthritis of the knee. Yonsei Med J 2012;53:801-5.

23. Hochman JR, Davis AM, Elkayam J, Gagliese L, Hawker GA. Neuropathic pain symptoms on the modified painDETECT correlate with signs of central sensitization in knee osteoarthritis. Osteoarthritis Cartilage 2013;21:1236-42.

24. Orita S, Ishikawa T, Miyagi M, Ochiai N, Inoue G, Eguchi $Y$, et al. Pain-related sensory innervation in monoiodoacetate-induced osteoarthritis in rat knees that gradually develops neuronal injury in addition to inflammatory pain. BMC Musculoskelet Disord 2011;12:134.

25. Bove SE, Laemont KD, Brooker RM, Osborn MN, Sanchez BM, Guzman RE, et al. Surgically induced osteoarthritis in the rat results in the development of both osteoarthritis-like joint pain and secondary hyperalgesia. Osteoarthritis Cartilage 2006;14:1041-8.

26. McDougall JJ, Andruski B, Schuelert N, Hallgrímsson $\mathrm{B}$, Matyas JR. Unravelling the relationship between age, nociception and joint destruction in naturally occurring osteoarthritis of Dunkin Hartley guinea pigs. Pain 2009;141:222-32.

27. Hochman JR, Gagliese L, Davis AM, Hawker GA. Neuropathic pain symptoms in a community knee OA cohort. Osteoarthritis Cartilage 2011;19:647-54.

28. Perrot S, Dieudé P, Pérocheau D, Allanore Y. Comparison of pain, pain burden, coping strategies, and attitudes between patients with systemic sclerosis and patients with rheumatoid arthritis: a crosssectional study. Pain Med 2013;14:1776-85.

29. Wu Q, Inman RD, Davis KD. Neuropathic pain in ankylosing spondylitis: a psychophysics and brain imaging study. Arthritis Rheum 2013;65:1494-503. 